\title{
Levels and factors of knowledge about the related health risks of exposure to secondhand smoke among medical students: A cross-sectional study in Jeddah, Saudi Arabia
}

\author{
Sami H. Alzahrani'
}

\begin{abstract}
INTRODUCTION Secondhand smoke (SHS) appears to be an insidious public health issue in Saudi Arabia, with a high percentage of people being reportedly exposed. In an attempt to raise awareness about this issue, we explored medical students' level of knowledge about SHS risks, as well as their levels of exposure to SHS and the correlation between knowledge and exposure.

METHODS A cross-sectional study was conducted in February 2020 at the Faculty of Medicine, King Abdulaziz University, Jeddah, Saudi Arabia. An online, modified version of a validated questionnaire was distributed among 2 nd to 6 th year medical students. The questionnaire mainly explored the following items: 1) exposure level to SHS; 2) impact of SHS exposure; and 3) knowledge about SHS related health risks, with calculation of a knowledge score (range: $0-8$ ). Adequate knowledge was defined as a score $\geq 5$ (median $=5$ ), and associations with sociodemographic and lifestyle factors and exposure to SHS were analyzed using chi-squared and independent t-test, as appropriate.

RESULTS Of a total 416 participants, $65.0 \%$ declared having one or more smoking persons among acquaintances, $26.4 \%$ being exposed to SHS at home, and $40.1 \%$ exposed to parental smoking during childhood. Further, $79.8 \%$ reported being regularly exposed to SHS in public places. Majority of participants reported significant discomfort (53.2\%) and physical symptoms (98.3\%) subsequent to SHS exposure. Among the list of 8 health risks, ear infection in children (28.1\%), heart attack in children (37.5\%), and cognitive deficit (47.8\%) were the least frequently identified. Adequate knowledge was found among $57.5 \%$ of the participants, and was higher among females and lower among participants living with their friends, compared to their counterparts $(\mathrm{p}<0.05)$. No association of knowledge level was observed with the parameters of exposure to SHS and poor discomfort feeling. CONCLusions The level of knowledge of medical students about health risks related to passive smoking is inadequate, while their exposure to SHS in public places is still substantial. This urges for the reinforcement of tobacco control strategies and highlights the great interest for medical colleges to implement effective educational interventions by improving their curricula regarding the risks of SHS and the benefits of smoking cessation.
\end{abstract}

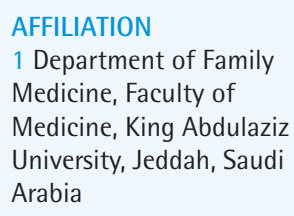

CORRESPONDENCE TO

Sami H. Alzahrani.

Department of Family

Medicine, Faculty of

Medicine, King Abdulaziz

University, P.O. Box 4828,

Jeddah 22431, Saudi Arabia.

E-mail:drsamihz@gmail.com

ORCID ID: https://orcid.

org/0000-0001-6786-7184

\section{KEYWORDS}

secondhand smoke, passive smoking, exposure, knowledge, awareness

Received: 10 June 2020 Revised: 7 October 2020 Accepted: 9 October 2020

\section{INTRODUCTION}

For a long time, smoking has been identified as a major risk factor of multiple illnesses and mortality worldwide $^{1}$, representing one of the greatest health epidemics in the twentieth century. Smokers are prone to a diverse array of negative consequences 
as indicated by the susceptibility to acute and longterm deleterious illnesses such as respiratory diseases, cancer, coronary heart disease, and deterioration in self-reported health, with subsequent school/work absenteeism and mortality ${ }^{2,3}$. Such consequences are complicated by the substantial prevalence of smoking. For example, in Saudi Arabia, the prevalence of smoking ranges between $13 \%$ and $38 \%$ among males, and this is supported by the significant rise of tobacco sales relative to the population size $e^{4,5}$. The pooled prevalence among college students has been estimated at $17 \%$, and it is higher in males than in females $(26 \% \text { versus } 5 \% \text {, respectively })^{6}$. Smoking has been a significant risk factor for cardiovascular diseases in the kingdom, and it is a forthcoming alarming threat for other tobacco-related illnesses, such as chronic obstructive pulmonary disease ${ }^{7}$.

However, the impact of the toxic effects of tobacco smoking is not only limited to active smokers, but also includes individuals exposed to secondhand smoke (SHS) or passive smokers. For instance, children of smoking parents are susceptible to short-term health risks, such as ear and respiratory diseases, as well as chronic diseases including inflammatory bowel disease and endothelial cell dysfunction ${ }^{8,9}$. Besides, the exposure to SHS has been associated with metabolic deficits, such as DNA and lipid oxidative damage. Additionally, individuals exposed to SHS have similar patterns of reduced antioxidant mechanisms as those observed in active smokers, such as reduced concentrations of vitamin $\mathrm{C}$ and vitamin $\mathrm{A}^{10}$. Consequently, SHS exposure is causally associated with lessened immune status, reduced lung functions, stroke, coronary heart disease, and other chronic diseases as in the case of active smoking ${ }^{11}$, and SHS-exposed children are more likely to experience leukemia, brain tumors, and lymphomas than nonexposed children ${ }^{1,12}$. Although the health risks of SHS are less frequently encountered than those in active smokers, the fact that the harmful effects are incurred even at very low doses indicates the lack of a specific safe threshold of exposure ${ }^{13}$. This emphasizes the need to address such a public health challenge.

The implications of these risks are significant, particularly in areas with a high prevalence of SHS exposure. In Saudi Arabia, despite a reinforced antismoking law banning smoking in public areas and explicitly stating the priority to protect non-smokers from exposure to tobacco smoke and emissions, particularly persons under 18 years of age $\mathrm{e}^{14}$, the issue of SHS is still a national concern. A nationwide study contributing to the Global Youth Tobacco Survey (GYTS) revealed that three in ten adolescents are exposed to SHS at home, and about $37.5 \%$ of students aged 13-15 years are exposed to smoke outdoors ${ }^{15}$.

Therefore, the role of physicians in treating tobacco dependence and raising public awareness about the health risks associated with smoking and SHS is more than ever critical. It has been shown that brief advice can markedly increase cessation rates ${ }^{16,17}$. However, there are multiple challenges associated with appropriate counselling. These include lack of self-efficacy and self-confidence in counselling skills, lack of proficiency with the methods of cessation and the risks of SHS exposure, lack of allocated time, and the lack of adequate cessation services integrated in healthcare systems ${ }^{18}$.

These challenges are compounded by the attitudes and levels of knowledge regarding smoking and SHS exposure among healthcare workers, and several local studies indicate a deficit in medical education curricula in tobacco dependence treatment and tobacco related topics, notably in undergraduate years ${ }^{18-20}$. On the other hand, the prevalence of smoking among medical students locally ranged from $13 \%$ to $28.9 \%^{21-23}$, and there is evidence of the role of peers in Saudi colleges as predictors of future active smoking ${ }^{5,24}$.

Indeed, these figures are alarming since healthcare providers, including medical students, should have adequate knowledge of SHS and be highly aware of its importance to educate patients, smokers and the public. Thus, we aimed at providing insight into the gaps in knowledge about SHS among medical students, and profiling the healthiness of their lifestyle and academic environment.

The present study assessed the level of knowledge of medical students regarding SHS-related health risks, and secondarily explored the associated factors with knowledge, as well as sources of information. Furthermore, it investigated the levels of exposure to SHS in the students' living and study environments and the impact of such exposure on somatic health and behavior.

\section{METHODS}

\section{Population and setting}

A cross-sectional study was conducted in February 
2020 among medical students at the Faculty of Medicine, King Abdulaziz University, Jeddah, Saudi Arabia. All undergraduate medical students from second to sixth year were considered eligible to participate. Students aged $<18$ years or $>30$ years were not included. The study was approved by the Institutional Review Board of King Abdulaziz University (Institutional Review Board 22-1-19). After obtaining ethical approval for the research from KAU, the email addresses of the selected students were obtained from Academic Affairs. The questionnaire was administered online via the SurveyMonkey platform (SurveyMonkey Europe UC), and the web link was sent via email to all selected participants, including a statement for explicit and written consent to participate. In addition, a notification was attached about the study objectives and response confidentiality. Potential participants were advised that the study results would be involved in a statistical analysis and aimed at being published in a peer-reviewed journal. The datasets used and/or analyzed during the current study are available from the author on reasonable request and all original data are available from the Department of Family Medicine, KAU, Jeddah, Saudi Arabia.

\section{Sampling}

Target sample size $(\mathrm{N}=332)$ was calculated to detect an unknown proportion (50\%) of students with adequate knowledge about SHS risk factors out of 2410 eligible medical students, with 5\% margin error and $95 \%$ confidence interval. This was calculated using Raosoft software (Raosoft, Seattle, WA, USA), available at: http://www.raosoft.com/samplesize.html. The target sample size was increased to $\mathrm{N}=480$ to compensate for eventual non-response, assuming $70 \%$ response rate.

The target population was stratified by academic level (2nd, 3rd, ..., year of studies) and a proportional allocation was used to randomly include participants based on the weighted percentage of students in each academic year. The sample sizes were: $25 \%(152 / 609)$ from 2 nd year, $20 \%$ (98/491) from 3rd year, $21 \%$ (106/507) from 4 th year, 17\% (72/424) from 5 th year, and $15 \%(57 / 379)$ from 6 th year.

\section{Questionnaire structure}

Permission was obtained from Lee et al. ${ }^{25}$ to use their questionnaire, as published in a study on SHS knowledge among hospital staff. An electronic, modified version of the questionnaire was adapted by not including the Fagerström test for nicotine dependence and some irrelevant (for Saudi Arabia) items such as alcohol consumption. The questionnaire covered five topics.

\section{Parameters of exposure to SHS}

These included: a number of smoking person categories among acquaintances including father, mother, brothers, sisters, friends, and other relatives, each accounting for one person-category; history of living with a smoking parent during childhood; places of SHS exposure, by answering the question 'where are you usually exposed to SHS?' with response options: at home - living room, at home - outside room, around the hospital, in the hospital, street corners, public places; and other exposure to SHS by any chance.

\section{Impact and consequences of exposure to SHS}

These included: perceived level of discomfort when exposed to SHS using a 5-point Likert-type discomfort scale (from 'feels good' to 'painful'); any somatic symptoms (nose and eyes irritation, respiratory symptoms, chest discomfort, any other symptom); coping strategy (asked them to refrain from smoking, moved away from smoke, did nothing, or smoked together).

\section{Awareness and knowledge about SHS health risks}

This was ascertained by a question on SHS related health risks that included lung cancer, heart diseases, cognitive deficits, low birthweight, ear infections in children, heart attack to children, allergies in children, and asthma in children. Answers to the awareness and knowledge question were evaluated using a 5-point Likert-type scale of agreement (from 'strongly agree' to 'strongly disagree').

\section{Sources of knowledge about SHS risks}

This was ascertained by a question on the different sources of knowledge about SHS risks with options that included newspapers, TV programs, public service announcement, smoking cessation education, and acquaintances such as relatives and friends, with responses provided as 'yes' or 'no'. 
Opinions regarding smoking prohibition in public places These included the need to make the prohibition compulsory, leaving it to the individual's personal conscience, or no prohibition at all.

Additionally, sociodemographic characteristics such as age, gender, marital status, GPA etc., and clinical and lifestyle data such as physical activity, history of chronic diseases, and smoking status, were collected.

\section{Statistical analysis}

After completion of the questionnaire, results were downloaded in a comma-separated values file format. The dataset was converted into an SPSS database (SPSS version 21.0 for Windows, SPSS Inc., Chicago, IL, USA). Descriptive statistics were used to summarize participants' sociodemographic and lifestyle characteristics as well as the pattern of answering to the different parts of the questionnaire. Knowledge subscale ( 8 items) was analyzed for reliability by calculating Cronbach's alpha. Knowledge score (range: $0-8$ ) was calculated as the number of health risks that were correctly identified by the participant, where 'strongly agree' or 'agree' were considered as a correct identification. The distribution of the variable (knowledge score) was analyzed using Kolmogorov-Smirnov and Shapiro-Wilk tests. Given non-normal distribution of knowledge score (see results), knowledge level was dichotomized into adequate and inadequate knowledge, using a knowledge score $\geq$ median to define adequate knowledge. Thus, the association of knowledge level (the dependent variable) with the independent variables including sociodemographic and lifestyle factors, parameters of exposure to SHS, and discomfort feeling regarding exposure and attitude towards prohibition of smoking in public places were analyzed using a chi-squared test for categorical variables, and independent t-test for continuous variables such as age. Multivariate binary regression was carried out using the 'enter' method to analyze the independent factors of adequate knowledge; results are presented as odds ratio (OR) with $95 \%$ confidence interval (95\% CI). In such a regression model, the level of knowledge was the dependent variable and the significantly associated factors were entered as independent variables. Secondarily, we analyzed the association of perceived level of discomfort with parameters of exposure to SHS including number of smoking persons among acquaintances and number of exposure places; we analyzed both discomfort score (range: 0-4) and percentage of participants with high level of discomfort $(\geq 3)$, using Kruskal-Wallis (nonparametric) test and chi-squared test, respectively. A p value $<0.05$ was considered to reject the null hypothesis.

\section{RESULTS}

\section{Participants' characteristics}

A total of 416 medical students replied to the questionnaire (response rate: $86.7 \%$ ); mean (SD) age 21.75 (1.60) years, $56.0 \%$ females, and $97.8 \%$ single. Distribution by academic level ranged from $10.3 \%$ for 3 rd year to $26.9 \%$ for 6 th year, and the majority had high $(38.9 \%)$ or average $(56.0 \%)$ grade point average (GPA). Clinical and lifestyle characteristics showed that $23.1 \%$ were overweight and $13.9 \%$ obese, $50.7 \%$ with low level of physical activity, and $12.3 \%$ having a chronic disease. Smoking status showed 13.9\% active smokers and $4.6 \%$ quitters, with the majority of active smokers $(63.8 \%)$ consuming $>10$ cigarettes daily (Table 1).

Table 1. Characteristics of medical students of the Faculty of Medicine, King Abdulaziz University $(\mathrm{N}=416)$

\begin{tabular}{llrl} 
Characteristics & Category & n \\
Sociodemographic & & & \\
\hline $\begin{array}{l}\text { Age (years), } \\
\text { mean } \pm \text { SD }\end{array}$ & & $21.75 \pm 1.60$ \\
Gender & Male & 183 & 44.0 \\
& Female & 233 & 56.0 \\
Marital status & Single & 407 & 97.8 \\
& Married & 9 & 2.2 \\
Nationality & Saudi & 398 & 95.7 \\
& Non-Saudi & 18 & 4.3 \\
Parents' marital & Married & 341 & 82.0 \\
status & Divorced & 33 & 7.9 \\
& Widow & 42 & 10.1 \\
Rank in siblings & Eldest & 88 & 21.2 \\
& Not the eldest & 328 & 78.8 \\
Monthly family & $<3000$ & 13 & 3.1 \\
income (SAR) & $3000-10000$ & 77 & 18.5 \\
& $>10000$ & 326 & 78.4 \\
Residence & Jeddah & 383 & 92.1 \\
& Other & 33 & 7.9 \\
& & & Continued \\
& & & \\
& & &
\end{tabular}


Table 1. Continued

\begin{tabular}{|c|c|c|c|}
\hline Characteristics & Category & $n$ & $\%$ \\
\hline \multirow[t]{3}{*}{ Housing } & Rental & 316 & 76.0 \\
\hline & Own house & 83 & 20.0 \\
\hline & Students dormitory & 17 & 4.1 \\
\hline \multirow[t]{3}{*}{ Living modality } & Alone & 381 & 91.6 \\
\hline & With family & 7 & 1.7 \\
\hline & With friends & 28 & 6.7 \\
\hline \multirow[t]{5}{*}{ Academic year } & 2nd & 94 & 22.6 \\
\hline & $3 r d$ & 43 & 10.3 \\
\hline & 4th & 94 & 22.6 \\
\hline & 5 th & 73 & 17.5 \\
\hline & 6th & 112 & 26.9 \\
\hline \multirow[t]{3}{*}{ GPA } & $\geq 4.5$ & 162 & 38.9 \\
\hline & $3.5-4.49$ & 233 & 56.0 \\
\hline & $2.5-3.49$ & 21 & 5.0 \\
\hline \multicolumn{4}{|l|}{$\begin{array}{l}\text { Lifestyle and } \\
\text { clinical }\end{array}$} \\
\hline \multirow[t]{6}{*}{$\mathrm{BMI}\left(\mathrm{kg} / \mathrm{m}^{2}\right)$} & Underweight (<18.5) & 49 & 11.8 \\
\hline & Normal (18.5-24.9) & 213 & 51.2 \\
\hline & Overweight (25.0-29.9) & 96 & 23.1 \\
\hline & Class I obesity (30.0-34.9) & 38 & 9.1 \\
\hline & Class II obesity (35.0-39.9) & 9 & 2.2 \\
\hline & Class III obesity ( $\geq 40.0$ ) & 11 & 2.6 \\
\hline \multirow{4}{*}{$\begin{array}{l}\text { Physical activity } \\
\text { (times per week) }\end{array}$} & 0 & 124 & 29.8 \\
\hline & 1 & 87 & 20.9 \\
\hline & $2-3$ & 40 & 9.6 \\
\hline & $>3$ & 165 & 39.7 \\
\hline \multirow[t]{7}{*}{ Chronic illness } & No & 365 & 87.7 \\
\hline & Yes & 51 & 12.3 \\
\hline & Asthma & 20 & 4.8 \\
\hline & Diabetes & 11 & 2.6 \\
\hline & $\begin{array}{l}\text { Chronic neurological } \\
\text { disease }\end{array}$ & 6 & 1.4 \\
\hline & Hypertension & 3 & 0.7 \\
\hline & Not specified & 13 & 3.1 \\
\hline \multirow[t]{3}{*}{ Smoking status } & Non-smoker & 339 & 81.5 \\
\hline & Quitter (>6 months) & 19 & 4.6 \\
\hline & Smoker & 58 & 13.9 \\
\hline \multirow{5}{*}{$\begin{array}{l}\text { Daily cigarettes } \\
\text { (among smokers) }\end{array}$} & $0-3$ & 13 & $3.1(22.4)$ \\
\hline & $4-10$ & 8 & $1.9(13.8)$ \\
\hline & $11-20$ & 18 & $4.3(31.0)$ \\
\hline & $>20$ & 7 & $1.7(12.1)$ \\
\hline & Not specified & 12 & $2.9(20.7)$ \\
\hline
\end{tabular}

SAR: 100 Saudi Riyal about 27 US\$. GPA: grade point average. SD: standard deviation.

\section{Secondhand smoke exposure, consequences and coping strategies}

In all, $75 \%$ of the participants declared having at least one smoking person among acquaintances, who was more frequently a relative $(38.5 \%)$, a friend $(34.6 \%)$, or the father $(26.7 \%)$. Further, $40.1 \%$ reported exposed to parental smoking during childhood. Regarding exposure places, majority reported being exposed to SHS in public places $(79.8 \%)$, while $26.4 \%$ reported being exposed at home, in room, and $24.0 \%$ outside room. On the other hand, $57.7 \%$ were regularly exposed to SHS in more than one place. Subsequent to exposure, $53.2 \%$ of the participants declared being very or extremely uncomfortable, 98.3\% reported experiencing physical symptoms such as chest discomfort $(27.9 \%)$, respiratory symptoms $(27.6 \%)$ and nose and eye irritations $(25.5 \%)$. The most frequently reported coping strategy regarding exposure to SHS was moving away from smoke (63.7\%), having a passive attitude $(21.4 \%)$, or asking smokers to refrain from smoking $(8.2 \%)$ (Table 2 ).

\section{Knowledge about secondhand smoke risks and attitude to smoking prohibition in public places} Ear infection in children $(28.1 \%)$, heart attack in children $(37.5 \%)$, and cognitive deficit $(47.8 \%)$ were

Table 2. Assessment of secondhand smoke exposure consequences and coping strategies among medical students of the Faculty of Medicine, King Abdulaziz University $(\mathrm{N}=416)$

\begin{tabular}{|c|c|c|c|}
\hline Type of exposure & Category & $n$ & $\%$ \\
\hline \multirow{8}{*}{$\begin{array}{l}\text { Smokers among } \\
\text { social circle/ } \\
\text { relatives }^{\mathrm{a}}\end{array}$} & Nobody & 104 & 25.0 \\
\hline & Yes (at least one person) & 312 & 75.0 \\
\hline & Father & 111 & 26.7 \\
\hline & Mother & 24 & 5.8 \\
\hline & Brother & 96 & 23.1 \\
\hline & Sister & 24 & 5.8 \\
\hline & Relative & 160 & 38.5 \\
\hline & Friend & 144 & 34.6 \\
\hline \multirow{5}{*}{$\begin{array}{l}\text { Number } \\
\text { of persons } \\
\text { smoking among } \\
\text { acquaintances }\end{array}$} & 0 & 104 & 25.0 \\
\hline & 1 & 165 & 39.7 \\
\hline & 2 & 78 & 18.8 \\
\hline & 3 & 46 & 11.1 \\
\hline & $\geq 4$ & 23 & 5.5 \\
\hline \multirow{2}{*}{$\begin{array}{l}\text { Having a smoking } \\
\text { parent during } \\
\text { childhood }\end{array}$} & No & 249 & 59.9 \\
\hline & Yes & 167 & 40.1 \\
\hline
\end{tabular}


Table 2. Continued

\begin{tabular}{|c|c|c|c|}
\hline Type of exposure & Category & $n$ & $\%$ \\
\hline \multirow{8}{*}{$\begin{array}{l}\text { Other exposure } \\
\text { by chance/ } \\
\text { place }(s)^{\mathrm{a}}\end{array}$} & No & 57 & 13.7 \\
\hline & Yes & 359 & 86.3 \\
\hline & At home, in room & 110 & 26.4 \\
\hline & At home, outside room & 100 & 24.0 \\
\hline & Around the hospital & 129 & 31.0 \\
\hline & In the hospital & 23 & 5.5 \\
\hline & Public places & 332 & 79.8 \\
\hline & Street corners & 147 & 35.3 \\
\hline \multirow{4}{*}{$\begin{array}{l}\text { Number of } \\
\text { places with SHS } \\
\text { exposure }\end{array}$} & $0-1$ & 176 & 42.3 \\
\hline & 2 & 118 & 28.4 \\
\hline & 3 & 79 & 19.0 \\
\hline & $\geq 4$ & 43 & 10.3 \\
\hline \multirow{5}{*}{$\begin{array}{l}\text { Feeling during } \\
\text { exposure }\end{array}$} & Good & 14 & 3.4 \\
\hline & Not uncomfortable & 67 & 16.1 \\
\hline & A bit uncomfortable & 114 & 27.4 \\
\hline & Very uncomfortable & 202 & 48.6 \\
\hline & Painful & 19 & 4.6 \\
\hline \multirow{5}{*}{$\begin{array}{l}\text { Symptoms arising } \\
\text { from exposure }^{\mathrm{a}}\end{array}$} & Nose and eyes irritation & 106 & 25.5 \\
\hline & Respiratory symptoms & 115 & 27.6 \\
\hline & Chest discomfort & 116 & 27.9 \\
\hline & Child respiratory & 15 & 3.6 \\
\hline & Any other symptom & 186 & 44.7 \\
\hline \multirow[t]{4}{*}{ Coping strategy } & $\begin{array}{l}\text { Asked them to refrain from } \\
\text { smoking }\end{array}$ & 34 & 8.2 \\
\hline & Moved away to avoid SHS & 265 & 63.7 \\
\hline & Did nothing & 89 & 21.4 \\
\hline & Smoked together & 28 & 6.7 \\
\hline
\end{tabular}

a More than one option possible.

the least frequently identified health risks of SHS among the list of eight; while lung cancer (84.9\%), asthma in children $(80.3 \%)$ and heart diseases (75.7\%) were the most frequently identified (Table $3)$. Reliability testing of the knowledge subscale showed a Cronbach alpha of 0.763 , indicating good internal consistency. Knowledge score showed a mean (SD) of 4.77 (2.01), median of 5 , and range of $0-8$; and normality testing showed KolmogorovSmirnov $($ statistic $=0.121, \mathrm{p}<0.0001)$; Shapiro-Wilk ( statistic $=0.956, \mathrm{p}<0.0001)$ concluding to nonnormally distributed variable (results not presented). Consequently, $57.5 \%$ of the participants had adequate knowledge, which was defined as a knowledge score $\geq 5$, the median being the cutoff. Acquaintances contributed to knowledge about SHS among $61.1 \%$ of the participants, while public service announcements and smoking cessation education contributed $44.0 \%$ and $38.9 \%$, respectively. Majority of the participants (64.4\%) thought that smoking should be strictly prohibited in public places while $9.4 \%$ advocated the right to smoke (Table 3 ).

\section{Factors associated with knowledge about secondhand smoke risks and attitude to secondhand smoke}

Knowledge about SHS-related health risks was greater in females of whom $63.5 \%$ had an adequate knowledge level (score $\geq 5$ ) versus $49.7 \%$ among males, and the difference was statistically significant $(p=0.005)$. Participants living with their friends had the lowest percentage of adequate knowledge (35.7\%), compared to their counterparts $(\mathrm{p}=0.044)$. No significant association of knowledge score was observed with

Table 3. Knowledge about secondhand smoke risks and attitude regarding smoking prohibition in public places among medical students of the Faculty of Medicine, King Abdulaziz University ( $N=416)$

\begin{tabular}{|c|c|c|c|}
\hline Parameter & Category & $n$ & $\%$ \\
\hline \multirow{2}{*}{$\begin{array}{l}\text { Awareness } \\
\text { about SHS } \\
\text { risks }\end{array}$} & Yes & 320 & 76.9 \\
\hline & No & 96 & 23.1 \\
\hline \multirow{8}{*}{$\begin{array}{l}\text { SHS-related } \\
\text { health risks } \\
\text { correctly } \\
\text { identified }\end{array}$} & Lung cancer & 353 & 84.9 \\
\hline & Heart diseases & 315 & 75.7 \\
\hline & Cognitive deficit & 199 & 47.8 \\
\hline & Low birthweight & 266 & 63.9 \\
\hline & Ear infection in children & 117 & 28.1 \\
\hline & Heart attack in children & 156 & 37.5 \\
\hline & Allergies in children & 243 & 58.4 \\
\hline & Asthma in children & 334 & 80.3 \\
\hline \multirow{2}{*}{$\begin{array}{l}\text { Knowledge } \\
\text { level }\end{array}$} & Inadequate (score <5) & 177 & 42.5 \\
\hline & Adequate (score $\geq 5$ ) & 239 & 57.5 \\
\hline \multirow{5}{*}{$\begin{array}{l}\text { Knowledge } \\
\text { source }^{\mathrm{a}}\end{array}$} & Newspapers & 37 & 8.9 \\
\hline & TV programs & 157 & 37.7 \\
\hline & Public service announcement & 183 & 44.0 \\
\hline & Smoking cessation education & 162 & 38.9 \\
\hline & Acquaintances & 254 & 61.1 \\
\hline \multirow{3}{*}{$\begin{array}{l}\text { What do you } \\
\text { think about } \\
\text { prohibiting } \\
\text { smoking } \\
\text { in public } \\
\text { places? }\end{array}$} & $\begin{array}{l}\text { lgnoring the right to smoke is } \\
\text { unfair }\end{array}$ & 39 & 9.4 \\
\hline & $\begin{array}{l}\text { Better leave it to each individual's } \\
\text { personal conscience }\end{array}$ & 109 & 26.2 \\
\hline & $\begin{array}{l}\text { It should be regulated more } \\
\text { strictly }\end{array}$ & 268 & 64.4 \\
\hline
\end{tabular}

a More than one option possible. 
the other sociodemographic and lifestyle factors, notably with smoking status ( $\mathrm{p}=0.181$ ) or amount of smoking $(p=0.912)$ (Table 4). Knowledge level was not associated with the number of persons smoking among acquaintances $(\mathrm{p}=0.392)$, having a smoking parent during childhood $(\mathrm{p}=0.831)$ and exposure by chance to SHS $(p=0.220)$. Similarly, no association of knowledge level was observed with level of discomfort feeling $(\mathrm{p}=0.342)$, coping strategy $(\mathrm{p}=0.706)$, and attitude toward smoking prohibition in public places $(\mathrm{p}=0.833)$ (Table 5).

The significantly associated variables were entered in a logistic regression model to further investigate their independent relationships with adequate knowledge. The regression model showed that adequate knowledge about SHS was independently associated with female

Table 4. Sociodemographic and clinical and lifestyle factors associated with knowledge about secondhand smoke risks as revealed in the study of medical students of the Faculty of Medicine, King Abdulaziz University $(\mathbf{N}=416)$

\begin{tabular}{|c|c|c|c|c|c|c|}
\hline \multirow[t]{3}{*}{ Factors } & \multirow[t]{3}{*}{ Category } & \multicolumn{4}{|c|}{ SHS knowledge level a } & \multirow[b]{3}{*}{$p$} \\
\hline & & \multicolumn{2}{|c|}{ Inadequate } & \multicolumn{2}{|c|}{ Adequate } & \\
\hline & & $n$ & $\%$ & $n$ & $\%$ & \\
\hline \multicolumn{7}{|l|}{ Sociodemographic } \\
\hline \multicolumn{2}{|l|}{ Age (years), mean \pm SD } & \multicolumn{2}{|c|}{$21.77 \pm 1.58$} & \multicolumn{2}{|c|}{$21.73 \pm 1.61$} & 0.820 \\
\hline \multirow[t]{2}{*}{ Gender } & Male & 92 & 50.3 & 91 & 49.7 & \\
\hline & Female & 85 & 36.5 & 148 & 63.5 & $0.005^{*}$ \\
\hline \multirow[t]{2}{*}{ Marital status } & Single & 173 & 42.5 & 234 & 57.5 & \\
\hline & Married & 4 & 44.4 & 5 & 55.6 & 1.000 \\
\hline \multirow[t]{2}{*}{ Nationality } & Saudi & 169 & 42.4 & 229 & 57.5 & \\
\hline & Non-Saudi & 8 & 44.4 & 10 & 55.6 & 0.868 \\
\hline \multirow[t]{3}{*}{ Parents' marital status } & Married & 142 & 41.6 & 199 & 58.4 & \\
\hline & Divorced & 17 & 51.5 & 16 & 48.5 & \\
\hline & Widow & 18 & 42.9 & 24 & 57.1 & 0.548 \\
\hline \multirow[t]{2}{*}{ Rank in siblings } & Eldest & 37 & 42.0 & 51 & 58.0 & \\
\hline & Not the eldest & 140 & 42.7 & 188 & 57.3 & 0.914 \\
\hline \multirow[t]{3}{*}{ Monthly family income (SAR) } & $<3000$ & 5 & 38.5 & 8 & 61.5 & \\
\hline & $3000-10000$ & 30 & 39.0 & 47 & 61.0 & \\
\hline & $>10000$ & 142 & 43.6 & 184 & 56.4 & 0.730 \\
\hline \multirow[t]{2}{*}{ Residence } & Jeddah & 158 & 41.3 & 225 & 58.7 & \\
\hline & Other & 19 & 57.6 & 14 & 42.4 & 0.069 \\
\hline \multirow[t]{3}{*}{ Housing } & Rental & 132 & 41.8 & 184 & 58.2 & \\
\hline & Own house & 36 & 43.4 & 47 & 56.6 & \\
\hline & Students dormitory & 9 & 52.9 & 8 & 47.1 & 0.653 \\
\hline \multirow[t]{3}{*}{ Living modality } & Alone & 157 & 41.2 & 224 & 58.8 & \\
\hline & With family & 2 & 28.6 & 5 & 71.4 & \\
\hline & With friends & 18 & 64.3 & 10 & 35.7 & $0.044^{*}$ \\
\hline \multirow[t]{5}{*}{ Academic year } & 2nd & 39 & 41.5 & 55 & 58.5 & \\
\hline & $3 r d$ & 15 & 34.9 & 28 & 65.1 & \\
\hline & 4th & 44 & 46.8 & 50 & 53.2 & \\
\hline & 5 th & 28 & 38.4 & 45 & 61.6 & \\
\hline & 6 th & 51 & 45.5 & 61 & 54.5 & 0.608 \\
\hline \multirow[t]{3}{*}{ GPA } & $<4.5$ & 63 & 38.9 & 99 & 61.1 & \\
\hline & $3.5-4.49$ & 107 & 45.9 & 126 & 54.1 & \\
\hline & $2.5-3.49$ & 7 & 33.3 & 14 & 66.7 & 0.259 \\
\hline
\end{tabular}


Table 4. Continued

\begin{tabular}{|c|c|c|c|c|c|c|}
\hline \multirow[t]{3}{*}{ Factors } & \multirow[t]{3}{*}{ Category } & \multicolumn{4}{|c|}{ SHS knowledge level a } & \multirow[b]{3}{*}{$p$} \\
\hline & & \multicolumn{2}{|c|}{ Inadequate } & \multicolumn{2}{|c|}{ Adequate } & \\
\hline & & $n$ & $\%$ & $n$ & $\%$ & \\
\hline \multicolumn{7}{|l|}{ Lifestyle and clinical } \\
\hline \multirow[t]{4}{*}{ BMI $\left(k g / m^{2}\right)$} & Underweight & 20 & 40.8 & 29 & 59.2 & \\
\hline & Normal & 86 & 40.4 & 127 & 59.6 & \\
\hline & Overweight & 44 & 45.8 & 52 & 54.2 & \\
\hline & Obese & 27 & 46.6 & 31 & 53.4 & 0.735 \\
\hline \multirow[t]{4}{*}{ Physical activity (times per week) } & 0 & 51 & 41.1 & 73 & 58.9 & \\
\hline & 1 & 42 & 48.3 & 45 & 51.7 & \\
\hline & $2-3$ & 16 & 40.0 & 24 & 60.0 & \\
\hline & $>3$ & 68 & 41.2 & 97 & 58.8 & 0.683 \\
\hline \multirow[t]{2}{*}{ Chronic illness } & No & 16 & 31.4 & 35 & 68.6 & \\
\hline & Yes & 161 & 44.1 & 204 & 55.9 & 0.085 \\
\hline \multirow[t]{3}{*}{ Smoking status } & Non-smoker & 137 & 40.4 & 202 & 59.6 & \\
\hline & Quitter (>6 months) & 10 & 52.6 & 9 & 47.4 & \\
\hline & Smoker & 30 & 51.7 & 28 & 48.3 & 0.181 \\
\hline \multirow[t]{5}{*}{ Daily cigarettes (among smokers) } & $0-3$ & 7 & 53.8 & 6 & 46.2 & \\
\hline & $4-10$ & 5 & 62.5 & 3 & 37.5 & \\
\hline & $11-20$ & 9 & 50.0 & 9 & 50.0 & \\
\hline & $>20$ & 4 & 57.1 & 3 & 42.9 & \\
\hline & Not specified & 5 & 41.7 & 7 & 58.3 & 0.912 \\
\hline
\end{tabular}

SAR: 100 Saudi Riyal about 27 US\$. GPA: grade point average. SD: standard deviation. ${ }^{*}$ Statistically significant result at $p<0.05$. a Inadequate score $<5$, adequate score $\geq 5$.

Table 5. Association of knowledge with exposure and attitude to secondhand smoke among medical students of the Faculty of Medicine, King Abdulaziz University ( $N=416)$

\begin{tabular}{|c|c|c|c|c|}
\hline \multirow[t]{2}{*}{ Type of exposure } & \multirow[t]{2}{*}{ Category } & \multicolumn{2}{|c|}{ Knowledge } & \multirow[b]{2}{*}{$p$} \\
\hline & & $\begin{array}{c}\text { Inadequate } \\
n(\%)\end{array}$ & $\begin{array}{c}\text { Adequate } \\
n(\%)\end{array}$ & \\
\hline \multirow{5}{*}{$\begin{array}{l}\text { Number of persons smoking } \\
\text { among acquaintances }\end{array}$} & 0 & 48 (46.2) & $56(53.8)$ & \\
\hline & 1 & $64(38.8)$ & $101(61.2)$ & \\
\hline & 2 & $35(44.9)$ & $43(55.1)$ & \\
\hline & 3 & $23(50.0)$ & $233(50.0)$ & \\
\hline & $\geq 4$ & 7 (30.4) & $16(69.6)$ & 0.392 \\
\hline \multirow{2}{*}{$\begin{array}{l}\text { Having a smoking parent during } \\
\text { childhood }\end{array}$} & No & $70(41.9)$ & $97(58.1)$ & \\
\hline & Yes & $107(43.0)$ & $142(57.0)$ & 0.831 \\
\hline \multirow[t]{2}{*}{ Exposure by chance to SHS } & No & $157(43.7)$ & $202(56.3)$ & \\
\hline & Yes & $20(35.1)$ & 37 (64.9) & 0.220 \\
\hline \multirow{4}{*}{$\begin{array}{l}\text { Number of places with SHS } \\
\text { exposure }\end{array}$} & $0-1$ & $87(49.4)$ & $89(50.6)$ & \\
\hline & 2 & $45(38.1)$ & 73 (61.9) & \\
\hline & 3 & $25(31.6)$ & $54(68.4)$ & \\
\hline & $\geq 4$ & $20(46.5)$ & $23(53.5)$ & $0.037^{*}$ \\
\hline \multirow{5}{*}{$\begin{array}{l}\text { Feeling during exposure } \\
\text { (discomfort score) }\end{array}$} & Good (0) & $7(50.0)$ & $7(50.0)$ & \\
\hline & Not uncomfortable (1) & $35(52.2)$ & $32(47.8)$ & \\
\hline & A bit uncomfortable (2) & $50(43.9)$ & $64(56.1)$ & \\
\hline & Very uncomfortable (3) & 78 (38.6) & $124(61.4)$ & \\
\hline & Painful (4) & 7 (36.8) & $12(63.2)$ & 0.342 \\
\hline
\end{tabular}


Table 5. Continued

\begin{tabular}{|c|c|c|c|c|}
\hline \multirow[t]{2}{*}{ Type of exposure } & \multirow[t]{2}{*}{ Category } & \multicolumn{2}{|c|}{ Knowledge } & \multirow[b]{2}{*}{$p$} \\
\hline & & $\begin{array}{c}\text { Inadequate } \\
n(\%)\end{array}$ & $\begin{array}{c}\text { Adequate } \\
n(\%)\end{array}$ & \\
\hline \multirow[t]{4}{*}{ Coping strategy } & Asked them to refrain from smoking & $13(38.2)$ & $21(61.8)$ & \\
\hline & Moved away to avoid SHS & $117(44.2)$ & $148(55.8)$ & \\
\hline & Did nothing & $34(38.2)$ & $55(61.8)$ & \\
\hline & Smoked together & $13(46.4)$ & $15(53.6)$ & 0.706 \\
\hline \multirow{3}{*}{$\begin{array}{l}\text { What do you think about } \\
\text { prohibiting smoking in public } \\
\text { places? }\end{array}$} & Ignoring the right to smoke is unfair & $15(38.5)$ & $24(61.5)$ & \\
\hline & Better leave it to each individual's conscience & $48(44.0)$ & $61(56.0)$ & \\
\hline & It should be regulated more strictly & $114(42.5)$ & $154(57.5)$ & 0.833 \\
\hline
\end{tabular}

* Statistically significant result at $p<0.05$

Table 6. Results of the multivariate binary logistic regression analysis of the predictors of adequate knowledge regarding SHS

$\begin{array}{llccc}\text { Parameter } & \text { Category } & \text { OR } & 95 \% \text { CI } & p \\ \text { Gender } & \text { Male } & \text { Ref. } & & \\ & \text { Female } & 1.628 & 1.082-2.450 & 0.019^{*} \\ \text { Living modality } & \text { Alone } & \text { Ref. } & & \\ & \text { With family } & 1.526 & 0.287-8.118 & 0.620 \\ & \text { With friends } & 0.432 & 0.189-0.987 & 0.046^{*} \\ \text { Number of } & 0-1 & \text { Ref. } & & \\ \text { places with SHS } & 2 & 1.576 & 0.969-2.562 & 0.067 \\ \text { exposure } & 3 & 2.156 & 1.224-3.799 & 0.008^{*} \\ & \geq 4 & 1.237 & 0.625-2.450 & 0.542\end{array}$

Cl: confidence interval. OR: odds ratio. ${ }^{*}$ Statistically significant result at $p<0.05$. gender $(\mathrm{OR}=1.63 ; 95 \% \mathrm{CI}: 1.08-2.45 ; \mathrm{p}=0.019)$, exposure to SHS in three places $(\mathrm{OR}=2.16$; 95\% CI: $1.22-3.80 ; \mathrm{p}=0.008)$, and living with friends $(\mathrm{OR}=0.43$; 95\% CI: 0.19-0.99; $\mathrm{p}=0.046$ ) (Table 6).

\section{Association between parameters of exposure to SHS and discomfort feeling}

Both mean discomfort level (Kruskal-Wallis test, $\mathrm{p}=0.004$ ) and percentage of participants with high level of discomfort (chi-squared, $\mathrm{p}=0.007$ ) were inversely correlated with the number of persons smoking among acquaintances. However, no association of the discomfort level was found with the number of exposure places (Figure 1).

Figure 1. Association between parameters of exposure to SHS and discomfort feeling
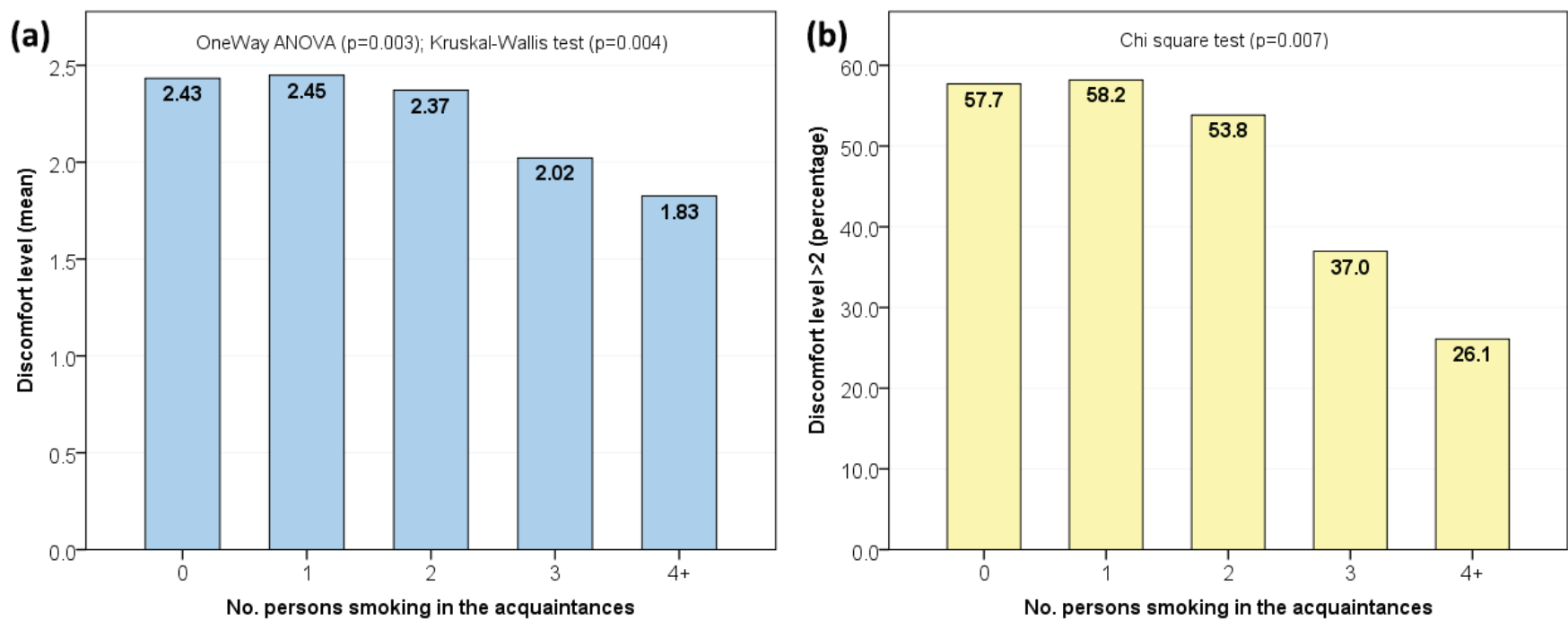
Figure 1. Continued
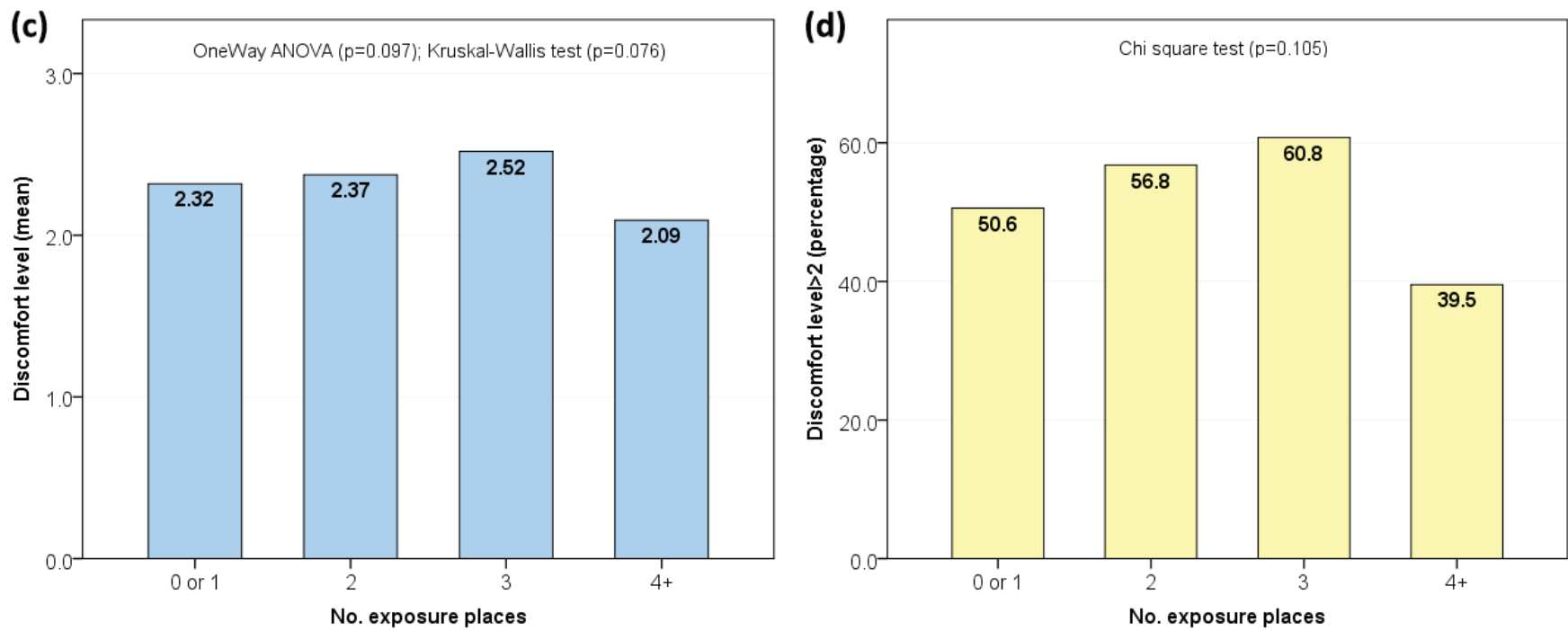

(a) Mean level of discomfort feeling (range: $0-4$ ) by number of persons smoking among acquaintances. (b) Percentage of participants with significant level of discomfort ( $>2$ ) by the number of persons smoking among acquaintances. (c) Mean level of discomfort feeling by number of exposure places. (d) Percentage of participants with significant level of discomfort by number of exposure places.

\section{DISCUSSION}

In the present study, we showed that more than half of the medical students in a Saudi medical college had adequate knowledge regarding SHS risks, and that approximately $86 \%$ were exposed to SHS with no significant correlation between the level of knowledge about SHS health consequences and exposure to SHS. On the other hand, knowledge scores were significantly higher among females and lower among those living with friends compared to their counterparts. Besides, acquaintances represented the major sources of knowledge. However, levels of knowledge about SHS were not impacted by smoking status.

Knowledge regarding the risks of SHS exposure is a key factor contributing to reducing exposure to the harms of smoke. Higher knowledge levels have been associated with more protective attitudes among the general public, such as opening windows, keeping children away from the smoking environment, or adopting smoke-free homes ${ }^{26,27}$. In the healthcare field, knowledge levels and attitudes regarding SHS are important determinants of educating and guiding patients on the potential health risks that include all age categories; these knowledge aspects could be promoted during the early academic life of health professionals.
The results presented in our study are in agreement with other investigations. Public places in Saudi Arabia represented the most frequent sites of SHS exposure as declared by $79.8 \%$ of medical students in the present study. Similarly, $57.7 \%$ of students $(n=805)$ at King Saud University, Riyadh, reported SHS exposure in public places ${ }^{28}$. Approximately $46.3 \%$ of medical students in Tabuk were exposed to SHS, and $68 \%$ emphasized the importance of implementing strategies to ban smoking in public places ${ }^{29}$. Furthermore, the exposure to SHS in public areas has been repeatedly demonstrated among school students and the general public ${ }^{30,31}$. Actually, this contradicts the national efforts that have been established in the kingdom. The World Health Organization's global Framework Convention for Tobacco Control (FCTC) was adopted by the Ministry of Health in Saudi Arabia in $2005^{32}$. Consequently, smoking is banned in educational, governmental, and transportation facilities, as well as healthcare institutions and other public places. The increased exposure to SHS in these places indicates significant deficiencies in the application of tobacco laws. Therefore, there is a need to enforce these legislations and apply strict measures to reduce the burden of smoking. In addition, it is imperative to limit the access to tobacco products through the prohibition of tobacco sales, which are 
inherent requirements of the FCTC.

Another important element in tobacco control programs is promoting knowledge levels among the general population. Medical students are the future leaders and actors of such interventions by providing correct information; hence, their knowledge and commitment are essential pillars for the national tobacco control strategy. The mean (SD) knowledge score of medical students in the present study was 4.77 (2.01), which corresponds to a scaled score of 59.6/100. In a cross-sectional study among 420 dental students attending King Abdulaziz University, Mansour ${ }^{33}$ revealed a higher knowledge score (88.3/100) regarding SHS health risks. Compared to the percentage of medical students with adequate knowledge in our study (57.5\%), a larger proportion (71.7\%) of medical students had adequate knowledge regarding the risks of SHS in three different medical schools in the Central, Western, and Southern regions of the kingdom ${ }^{18}$. Beyond the discernable variances between these results and the differences in tools that were used, knowledge levels remain relatively low with regard to the studied medical student population. As a result, further interventions are required to enhance students' knowledge and to identify the predictors of poor knowledge.

In general, it is necessary to target medical students to increase their knowledge about the risks of smoking and consequences of SHS exposure. In a case control study, a group of medical students received an educational intervention comprising online video lectures to promote their knowledge and competency to manage patients with SHSrelated disorders ${ }^{34}$. Although knowledge scores were generally low for all recruited participants, students who participated in the educational intervention had significant changes in their pre-test and posttest scores. Besides, the group exposed to the SHS intervention reported a significant intent to screen patients at risk in each primary care examination. In India, the implementation of a tobacco intervention training program among first year medical students promoted knowledge levels and attitudes regarding smoking cessation and $\mathrm{SHS}^{35}$. Indeed, this would be reflected in their perceptions about future medical practices. About $77.4 \%$ of medical students in Riyadh believed that smoking healthcare professionals are less likely to advise their patients to quit smoking ${ }^{28}$.
Therefore, students at medical colleges have strong perceptions about the implications of healthcare professionals as role models to guide the community and the general population. Such behavior would be further supported as the students progress to their professional life.

In our study, females had higher knowledge levels regarding SHS. Lee et al. ${ }^{25}$ found similar results among hospital staff in a medical institution in South Korea. Interestingly, all surveyed females in such a study were non-smokers. Females were also significantly knowledgeable in other studies conducted in Pakistan ${ }^{36}$ and Jordan ${ }^{37}$. Supposedly, females would be more aware of SHS risks because they are more concerned with the adverse effects of environmental tobacco smoke on pregnancies and fetuses $^{38}$. This suggests that future interventions should primarily focused on male healthcare professionals and/or medical students. Notably, the influence of friends may be highlighted, which can be identified from a lower knowledge level about SHS among students living with friends (away from the family circle) as indicated in the results of the multivariate regression analysis in our study. This is further marked in the case when close friends are smokers, which is considered a major risk factor of SHS exposure outside the household ${ }^{31,39}$. Seemingly, male students with smoking friends have little information about SHS risks, and so they are more likely to express neutral attitudes towards smoking ${ }^{40,41}$. The influence of friends increases also the likelihood of future smoking, and having a non-smoking friend reduces that risk ${ }^{42-44}$. Cognitive vulnerabilities towards smoking uptake and SHS-related risks are influenced by peers early in children aged $9-10$ years $^{41}$. The friend effect could therefore be targeted in future intervention programs, and medical students could play a significant role to advise their friends to quit smoking.

In our study, the increased number of smokers among acquaintances has significantly reduced the participants' discomfort levels, whereas the number of exposure places did not alter the discomfort variables. These unexpected findings may indicate an increased level of social tolerance to SHS exposure, probably leading to inability of participants to identify SHS exposure in certain instances and inaccurate reporting of SHS exposure. Another aspect of social tolerance 
is the findings about coping strategy showing the majority $(>85 \%)$ reacting to SHS by passive avoidance or non-action, while only $8.2 \%$ declared opposing smoking in restricted places. This tolerance could be associated with high prevalence of smoking in some areas as revealed previously ${ }^{45}$. The impact of social tolerance is detrimental, as it causes significant harms to the exposed non-smokers and could represent a barrier to smoke-free policies. Thus, medical students should be more aware about this issue to contribute more effectively in patient education about the risks of SHS exposure, both among smokers and potential passive smokers.

\section{Limitations}

The present study has some limitations. The crosssectional design might have resulted in a significant bias in reporting distinct variables, such as the exposure to SHS and discomfort levels, which were measured using indirect and subjective indicators. Additionally, the outcomes of our analysis might not be fully representative of other Saudi medical colleges across the kingdom. This might be compounded by the differences in medical curricula at other national colleges. However, we believe that the results of the present study could pave the way for the implementation of robust educational interventions that target future doctors. This would ultimately be reflected in the national efforts of tobacco control within healthcare institutions to promote public health awareness.

\section{CONCLUSIONS}

Medical students in Jeddah, Saudi Arabia, had relatively low knowledge regarding the health risks of SHS exposure, particularly when compared to other medical colleges in the kingdom. While female students had higher knowledge levels than males, the friends' circle appears to play a significant role in awareness and knowledge about the health risks of SHS. There is great interest for medical colleges to implement effective educational interventions by improving their curricula regarding the risks of SHS and the benefits of smoking cessation. Notwithstanding the efforts of the Saudi Ministry of Health to ban smoking according to the FCTC treaty, the exposure to SHS in public places is still substantial. Therefore, it is crucial to reinforce tobacco control strategies and to increase taxation of tobacco products to support local legislations.

\section{REFERENCES}

1. US National Center for Chronic Disease Prevention and Health Promotion - Office on Smoking and Health. The Health Consequences of Smoking-50 Years of Progress: A Report of the Surgeon General. Atlanta, GA: US Centers for Disease Control and Prevention; 2014. https://www. ncbi.nlm.nih.gov/books/NBK179276/. Accessed June $10,2020$.

2. Onor IO, Stirling DL, Williams SR, et al. Clinical Effects of Cigarette Smoking: Epidemiologic Impact and Review of Pharmacotherapy Options. Int J Environ Res Public Health. 2017;14:1147. doi:10.3390/ijerph14101147

3. Creamer MR, Wang TW, Babb S, et al. Tobacco Product Use and Cessation Indicators Among Adults United States, 2018. MMWR Morb Mortal Wkly Rep. 2019;68:1013-1019. doi:10.15585/mmwr.mm6845a2

4. Salama R. Challenges facing smokers in Saudi Arabia. Int J Health Sci (Qassim). 2012;6(2):V-VI. doi:10.12816/0005986

5. Al-Zalabani A, Kasim K. Prevalence and predictors of adolescents' cigarette smoking in Madinah, Saudi Arabia: a school-based cross-sectional study. BMC Public Health. 2015;15:17. doi:10.1186/s12889-015-1363-8

6. Alotaibi SA, Alsuliman MA, Durgampudi PK. Smoking tobacco prevalence among college students in the Kingdom of Saudi Arabia: Systematic review and meta-analysis. Tob Induc Dis. 2019;17(April). doi:10.18332/tid/105843

7. Tahir M. Smoking and its risks in Saudi Arabia: Literature review. Hamdan Medical Journal. 2019;12(4): doi:10.4103/hmj.Hmj_7_18

8. Chivese T, Esterhuizen TM, Basson AR, Watermeyer G. The Influence of Second-Hand Cigarette Smoke Exposure during Childhood and Active Cigarette Smoking on Crohn's Disease Phenotype Defined by the Montreal Classification Scheme in a Western Cape Population, South Africa. PLoS One. 2015;10(9):e0139597. doi:10.1371/journal.pone.0139597

9. Messner B, Bernhard D. Smoking and cardiovascular disease: mechanisms of endothelial dysfunction and early atherogenesis. Arterioscler Thromb Vasc Biol. 2014;34:509-515. doi:10.1161/ATVBAHA.113.300156

10. Wilson KM, Finkelstein JN, Blumkin AK, Best D, Klein JD. Micronutrient levels in children exposed to secondhand tobacco smoke. Nicotine Tob Res. 2011;13:800-808. doi: $10.1093 / \mathrm{ntr} / \mathrm{ntr} 076$

11. US Office on Smoking and Health. The Health Consequences of Involuntary Exposure to Tobacco Smoke: A Report of the Surgeon General. Atlanta, GA: US Centers for Disease Control and Prevention US; 2006.

12. Mustafa Kamal N, Othman Abdul Qadir M, Ibrahim Hama Rahim B, M. Ibrahem A, Karadaghy B. Epidemiology and Risk Factors of Cancer Among Children and Adolescents 
in Sulaimani City: A Case-Control Study. Acta Med Iran. 57(3):184-193. doi:10.18502/acta.v57i3.1822

13. Committee on the Public Health Implications of Raising the Minimum Age for Purchasing Tobacco Products, Board on Population Health and Public Health Practice, Institute of Medicine. Public Health Implications of Raising the Minimum Age of Legal Access to Tobacco Products. Bonnie RJ, Stratton K, Kwan LY, eds. Washington, DC: National Academies Press; 2015. doi:10.17226/18997

14. Ministry of Health of Saudi Arabia. The Executive Regulations of Anti-Smoking Law issued by Royal Decree No. (M/56), dated 28/07/1436H (the 'Law'). https:// www.moh.gov.sa/en/Ministry/Rules/Documents/AntiTobacco-Executive-Regulations.pdf. Published 2015. Accessed August 5, 2020.

15. World Health Organization, US Centers for Disease Control and Prevention. Saudi Arabia - Global Youth Tobacco Survey 2010. https://extranet.who.int/ ncdsmicrodata/index.php/catalog/341/study-description. Accessed December 11, 2019.

16. Stead LF, Buitrago D, Preciado N, Sanchez G, HartmannBoyce J, Lancaster T. Physician advice for smoking cessation. Cochrane Database Syst Rev. 2013;(5). doi:10.1002/14651858.CD000165.pub4

17. 2008 PHS Guideline Update Panel, Liaisons, and Staff. Treating tobacco use and dependence: 2008 update U.S. Public Health Service Clinical Practice Guideline executive summary. Respir Care. 2008;53(9):1217-1222. PMID:18807274

18. Jradi H, Al-Shehri A. Knowledge about tobacco smoking among medical students in Saudi Arabia: findings from three medical schools. J Epidemiol Glob Health. 2014;4:269-276. doi:10.1016/j.jegh.2014.04.001

19. Sabra A, Taha A. Shisha Smoking: Knowledge and Perception among Male Medical University Students in Dammam City, Saudi Arabia. Journal of High Institute of Public Health. 2011;41:33-49. doi:10.21608/jhiph.2011.20144

20. Al-Jdani S, Mashabi S, Alsaywid B, Zahrani A. Smoking cessation counseling: Knowledge, attitude and practices of primary healthcare providers at National Guard Primary Healthcare Centers, Western Region, Saudi Arabia. J Family Community Med. 2018;25:175-182. doi:10.4103/jfcm.JFCM_142_17

21. Mahmoud AE. Prevalence of cardiovascular disease risk factors among Egyptian and Saudi medical students: a comparative study. J Egypt Public Health Assoc. 2015;90:3539. doi:10.1097/01.EPX.0000460969.93981.c2

22. Al-Turki YA. Smoking habits among medical students in Central Saudi Arabia. Saudi Med J. 2006;27:700-703. PMID:16680263

23. Wali SO. Smoking habits among medical students in Western Saudi Arabia. Saudi Med J. 2011;32:843-848. PMID:21858395

24. Almutairi KM. Predicting Relationship of Smoking Behavior Among Male Saudi Arabian College Students
Related to Their Religious Practice. J Relig Health. 2016;55:469-479. doi:10.1007/s10943-015-0003-z

25. Lee SR, Cho AR, Lee SY, et al. Secondhand smoke knowledge, sources of information, and associated factors among hospital staff. PLoS One. 2019;14:e0210981. doi:10.1371/journal.pone.0210981

26. Dunn J, Greenbank S, McDowell M, et al. Community knowledge, attitudes and behaviours about environmental tobacco smoke in homes and cars. Health Promot J Austr. 2008;19:113-117. doi:10.1071/he08113

27. Evans KA, Sims M, Judge K, Gilmore A. Assessing the knowledge of the potential harm to others caused by second-hand smoke and its impact on protective behaviours at home. J Public Health (Oxf). 2012;34:183194. doi:10.1093/pubmed/fdr104

28. Almutairi KM. Prevalence of tobacco use and exposure to environmental tobacco smoke among saudi medical students in Riyadh, Saudi Arabia. J Community Health. 2014;39:668-673. doi:10.1007/s10900-014-9900-4

29. Alshehri R, Alwakeel A, Alatawi O, et al. Pattern, knowledge, and attitude of smoking among medical students in Saudi Arabia. International Journal of Medicine in Developing Countries. 2019;3:441-445. doi:10.24911/ijmdc.51-1546557742

30. Moradi-Lakeh M, El Bcheraoui C, Tuffaha M, et al. Tobacco consumption in the Kingdom of Saudi Arabia, 2013: findings from a national survey. BMC Public Health. 2015;15. doi:10.1186/s12889-015-1902-3

31. Al-Zalabani AH, Amer SM, Kasim KA, Alqabshawi RI, Abdallah AR. Second-Hand Smoking among Intermediate and Secondary School Students in Madinah, Saudi Arabia. Biomed Res Int. 2015;2015. doi:10.1155/2015/672393

32. Munif M. Report on Tobacco Control Program of Ministry of Health In Saudi Arabia. http://www.sa-tcp.com/ newsite/user/pdf/REPORT_ON_TCP.pdf. Published 2009. Accessed December 14, 2019

33. Mansour AY. Predictors of Smoking among Saudi Dental Students in Jeddah. Am J Health Behav. 2017;41:329337. doi:10.5993/AJHB.41.3.12

34. Merlo LJ, Sutton JA, Gold MS. Brief educational intervention to improve medical student competence in managing patients exposed to secondhand smoke. Subst Abus. 2014;35:163167. doi:10.1080/08897077.2013.821438

35. Pati S. Putting tobacco cessation and prevention into undergraduate medical education. Int J Prev Med. 2014;5(1):69-75. PMID:24554994

36. Bhanji S, Andrades M, Taj F, Khuwaja AK. Factors related to knowledge and perception of women about smoking: a cross sectional study from a developing country. BMC Womens Health. 2011;11. doi:10.1186/1472-6874-11-16

37. Gharaibeh H, Haddad L, Alzyoud S, El-Shahawy O, Baker NA, Umlauf M. Knowledge, attitudes, and behavior in avoiding secondhand smoke exposure among nonsmoking employed women with higher education in Jordan. Int J Environ Res Public Health. 2011;8:4207- 
4219. doi:10.3390/ijerph8114207

38. Al-Shaikh GK, Alzeidan RA, Mandil AM, Fayed AA, Marwa B, Wahabi HA. Awareness of an obstetric population about environmental tobacco smoking. J Family Community Med. 2014;21:17-22. doi:10.4103/2230-8229.128768

39. Raute LJ, Pednekar MS, Mistry R, Gupta PC, Pimple SA, Shastri SS. Determinants of exposure to second-hand smoke at home and outside the home among students aged 11-17 years: results from the Mumbai Student Tobacco Survey 2010. Indian J Cancer. 2012;49:419-424. doi:10.4103/0019-509X.107750

40. Xu X, Chen C, Abdullah AS, Sharma M, Liu H, Zhao Y. Knowledge about and sources of smoking-related knowledge, and influencing factors among male urban secondary school students in Chongqing, China. Springerplus. 2016;5(1). doi:10.1186/s40064-016-3589-z

41. McGee CE, Trigwell J, Fairclough SJ, et al. Influence of family and friend smoking on intentions to smoke and smoking-related attitudes and refusal self-efficacy among 9-10 year old children from deprived neighbourhoods: a cross-sectional study. BMC Public Health. 2015;15:225. doi:10.1186/s12889-015-1513-z

42. Penzes M, Czegledi E, Balazs P, Foley KL. Factors associated with tobacco smoking and the belief about weight control effect of smoking among Hungarian adolescents. Cent Eur J Public Health. 2012;20:11-17. doi:10.21101/cejph.a3726

43. Mak KK, Ho SY, Day JR. Smoking of parents and best friend--independent and combined effects on adolescent smoking and intention to initiate and quit smoking. Nicotine Tob Res. 2012;14:1057-1064. doi:10.1093/ntr/nts008

44. Alves RF, Precioso J, Becona E. Smoking behavior and secondhand smoke exposure among university students in northern Portugal: Relations with knowledge on tobacco use and attitudes toward smoking. Pulmonology. 2020. doi:10.1016/j.pulmoe.2020.03.004

45. Jeong BY, Lim MK, Yun EH, Oh JK, Park EY, Lee DH. Tolerance for and potential indicators of second-hand smoke exposure among nonsmokers: a comparison of self-reported and cotinine verified second-hand smoke exposure based on nationally representative data. Prev Med. 2014;67:280-287. doi:10.1016/j.ypmed.2014.07.003
ACKNOWLEDGEMENTS

The author is thankful to the medical students of King Abdulaziz University for their participation in this study.

\section{CONFLICTS OF INTEREST}

The author has completed and submitted an ICMJE form for disclosure of potential conflicts of interest. The author declares that he has no competing interests, financial or otherwise, related to the current work. S.H. Alzahrani reports grants from King Abdulaziz University, during the conduct of the study.

\section{FUNDING}

This work was funded by the Deanship of Scientific Research (DSR), KAU, Jeddah, under grant No. DF-349-140-1441. The author acknowledges DSR with thanks for their technical and financial support. The funders had no role in study design, data collection and analysis, decision to publish, or preparation of the manuscript.

PROVENANCE AND PEER REVIEW

Not commissioned; externally peer reviewed. 